\title{
Characterization of Multi-GNSS Between-Receiver Differential Code Biases Using Zero and Short
}

\section{Baselines}

Baocheng Zhang $^{1 *}$ and Peter J.G. Teunissen ${ }^{1,2}$

${ }^{1}$ GNSS Research Centre, Curtin University, Australia

${ }^{2}$ Geoscience and Remote Sensing, Delft University of Technology, The Netherlands

*Corresponding author: b.zhang@curtin.edu.au; Tel: 61-8-9266-7566; Fax: 61-8-9266-2703

\begin{abstract}
Care should be taken to minimize adverse impact of receiver Differential Code Biases (DCBs) on Global Navigation Satellite System (GNSS) derived ionospheric parameters. It is therefore of importance to ascertain the intrinsic characteristics of receiver DCBs, preferably in the context of new generation GNSS. In this contribution we present a method that enables time-wise retrieval of Between-Receiver DCBs (BR-DCBs) from dual-frequency, code-only measurements collected by a pair of co-located receivers. This method is applicable to the US GPS as well as to a new set of GNSS constellations including the Chinese BeiDou, the European Galileo and the Japanese QZSS. With the use of this method, we determine the multi-GNSS BR-DCB time-wise estimates covering a time period of up to 2 years (Jan. 2013-Mar. 2015) with a 30 second time resolution for five receiver-pairs (four zero and one short baselines). For the BR-DCB time-wise estimates pertaining to an arbitrary receiver-pair and constellation, we demonstrate their promising intra-day stability by means of statistical hypothesis testing. We also find that the BeiDou BR-DCB daily weighted average (DWA) estimates show a dependence on satellite type, in particular for receiver-pairs of mixed types. Finally, we demonstrate that long-term variability in BR-DCB DWA estimates can be closely associated with hardware temperature variations inside the receivers.
\end{abstract}

\section{Keywords}

Global Navigation Satellite System (GNSS) . Total Electron Content (TEC) . Between-Receiver Differential Code Bias (BR-DCB) . BeiDou Code Inter-Satellite-Type-Bias (ISTB)

\section{Introduction}

It has long been recognized that the vertical Total Electron Content (vTEC) parameters determined from Global Navigation Satellite System (GNSS) measurements are quite beneficial to ionospheric studies [1-4]. Since June 1998, the International GNSS Service (IGS) ionosphere working group has started to routinely deliver the Global Ionosphere Maps (GIMs) in support of a wide range of atmospheric and geodetic applications [5-7]. Instead of relying almost exclusively on the US Global Positioning System (GPS), as was often the case throughout the past several decades [8-11], the emergence of new GNSS constellations in recent years would bring unprecedented opportunities for more detailed ionospheric investigations [12-14]. As of this writing, the Chinese BeiDou constellation is comprised of five Geostationary Orbit (GEO) satellites, five Inclined Geosynchronous Orbit (IGSO) satellites and four Medium Earth Orbit (MEO) satellites [15]. The ability of the BeiDou constellation to provide Positioning, Navigation and Timing (PNT) services in at least the Asia-Pacific region commenced in December 2012 [16]. The European Galileo has already completed the In-Orbit-Validation (IOV) phase and its constellation consists of four operational MEO satellites [17]. The Japanese Quasi-Zenith Satellite System (QZSS) 
is designed as a regional augmentation system for GPS [18]. The first operational QZSS satellite (IGSO) was launched in September 2010. Two additional IGSO satellites and one GEO satellite will be deployed into the orbit by the late 2010s [19].

The lumped effect of satellite and receiver Differential Code Biases (DCBs) is generally considered a major source of error in determination of vTEC [20]. In fact, satellite DCBs have been found to remain fairly stable over considerable periods of time for different GNSS constellations [21, 22]. This enables us to retrieve the satellite DCB estimates with rather high accuracy, particularly under calm ionospheric conditions [23, 24]. After that, removal of the effect of the satellite DCBs on vTEC determination would become simple and straightforward. For receiver DCBs, however, their variability may be evident even in a comparatively short period of time say one day or a couple of hours $[25,26]$. One of the main reasons for this is commonly identified as temperature perturbations around the receivers [27]. Hence, handling the short-term temporal variability of receiver DCBs is a very crucial task in order to enhance the reliability of GNSS-derived vTEC parameters.

Up to now, studies that have examined the receiver DCB characteristics using real GNSS data (albeit GPSonly) can be classified into two distinct groups. Researchers in the first group have focused their attentions on analyzing the receiver DCB estimates that are byproducts of vTEC determination [28-30]. Actually, these estimates with daily time resolution may be subject to severe modeling errors, originating mainly from the imperfection of vTEC mathematical representations. Just for this reason one might misleadingly assign the ionospheric variability as the primary cause of the receiver DCB estimate variations [28, 31]. Fortunately, this problem is avoidable in the second group of studies as it has completely got rid of the reliance on vTEC modeling process [25, 26, 32]. The basic procedure followed here is to first obtain the ionospheric observables, interpreted as line-of-sight ionospheric delays biased by the satellite and the receiver DCBs, for a pair of co-located receivers using so called carrier-tocode leveling process [33]. After taking the between-receiver difference of the ionospheric observables, for each satellite it will yield a series of Between-Receiver DCB (BR-DCB) time-wise estimates. The maximum spread between the BR-DCB time series corresponding to different satellites, which should ideally be zero, is finally treated as a diagnostic measure for inferring the variability of BR-DCB estimates. Nevertheless, this group of studies still has one significant disadvantage, namely, that the leveling errors underlying the ionospheric observables that are receiver/antenna dependent may corrupt the BR-DCB time-wise estimates [25].

Without the necessity of vTEC modeling or ionospheric observable formation, we propose in this contribution a time-wise BR-DCB retrieval method employing code-only measurements collected by a zero or short baseline from not only the GPS, but the BeiDou, Galileo and QZSS constellations as well. We take the between-receiver and between-frequency differences of these code measurements so as to minimize the error sources in retrieval of multi-GNSS BR-DCBs. We diagnose the intra-day stability of the BR-DCB time-wise estimates for different GNSS constellations and receiver-pairs using the statistical hypothesis testing scheme that takes into account the formal uncertainties of these estimates. Special care has been taken to properly deal with the code Inter-Satellite-Type-Biases (ISTBs) when retrieving BeiDou BR-DCBs with our method. Generally, the BeiDou code ISTBs interpreted as the 'double-differenced' receiver code biases between two receivers and two BeiDou satellite types are found to be significant for receiver-pairs of mixed types [34]. We are about to investigate 
the effect of them on the consistency between BeiDou GEO/IGSO/MEO BR-DCB estimates. With the fact that the receiver DCB temperature dependence is likely due to three factors (the antenna, the cable and the receiver hardware) in mind [27], we attempt to discern which one of them is the most influential based on a number of dedicatedly designed experiments.

\section{Methods}

In a rather compact vector-matrix form, we write the function model of our BR-DCB retrieval method as

$$
E\{\mathbf{P}(i)\}=\mathbf{e}_{m} b(i)
$$

with $E\{\cdot\}$ denoting the expectation operator and where $i$ denotes the epoch index. $m$ equals the total number of satellites that belong to one common GNSS constellation. The $m \times 1$ vector $\mathbf{e}_{m}$ has all 1's as its entries. The $m \times 1$ vector $\mathbf{P}(i)$ contains the between-receiver, between-frequency differenced code measurements and $b(i)$ is the unknown BR-DCB parameter.

For the entries of the diagonal variance matrix of $\mathbf{P}(i)$ we make use of an elevation-dependent weighting

$$
D\{\mathbf{P}(i)\}=\operatorname{diag}\left\{\frac{4 \sigma^{2}}{\sin ^{2}\left[\theta^{1}(i)\right]} \cdots \frac{4 \sigma^{2}}{\sin ^{2}\left[\theta^{m}(i)\right]}\right\}
$$

in which $D\{\cdot\}$ is the dispersion operator. $\sigma$ denotes the zenith-referenced undifferenced code standard deviation and $\theta^{s}(i)$ is the elevation angle of satellite $s=1, \cdots, m$ at epoch $i$.

Assuming that a pair of co-located receivers has collected code measurements from multiple GNSS constellations on at least two frequencies, one would then be able to get the least squares estimates of $b(i)$ (written as $\hat{b}(i))$ on an epoch-by-epoch and constellation-by-constellation basis with the use of Eqs. (1) and (2). Since the redundancy is defined as the number of observations minus the number of parameters, the single-epoch redundancy of our model is thus $m-1$. This implies that time-wise BR-DCB retrieval with our model is even possible for QZSS which for the time being is still a constellation of one satellite.

To make it clear whether the $\widehat{b}(i)$ exhibits a statistically significant change over a given period of time (1 day for instance), we construct a test statistic as follows

$$
T=\sum_{i=1}^{t} \frac{[\widehat{b}(i)-\bar{b}]^{2}}{\sigma_{\widehat{b}}^{2}(i)}
$$

where $t$ is the total number of epochs. $\bar{b}$ and $\sigma_{\widehat{b}}^{2}(i)$ denote, respectively, the daily weighted average (DWA) and formal uncertainty of $\widehat{b}(i)$. Test statistic $T$ would be Chi-squared distributed with $t-1$ freedom degrees if the $\widehat{b}(i)$ has been proven to be normally distributed. One would confirm the intra-day stability in $\widehat{b}(i)$ if $T<\chi_{\alpha}^{2}(t-1,0)$ occurs for a pre-specified significance level $\alpha$.

As we mentioned earlier, BeiDou BR-DCB retrieval needs to take care of the code ISTBs. To illustrate this point, we derive the following identity on the basis of the code ISTB definition described in [34] 


$$
d_{1}^{\beta \gamma}-d_{2}^{\beta \gamma}=\bar{b}^{\beta}-\bar{b}^{\gamma}
$$

with $\beta$ and $\gamma$ denoting two BeiDou satellite types. $d_{j}^{\beta \gamma}$ with $j=1,2$ are code ISTBs at two frequencies. $\bar{b}^{\beta}$ and $\bar{b}^{\gamma}$ are BR-DCB DWA estimates retrieved, respectively, using type- $\beta$ and type- $\gamma$ satellites' measurements. One can easily see from Eq. (4) that, $\bar{b}^{\beta}$ and $\bar{b}^{\gamma}$ would be essentially different if $d_{1}^{\beta \gamma}$ deviates substantially from $d_{2}^{\beta \gamma}$. We shall verify this finding later by retrieving the satellite type dependent BeiDou BR-DCB estimates for a common receiver-pair and then checking their consistency.

\section{Results}

\subsection{Field Campaign}

We deployed six multi-GNSS receivers, including three Trimble NETR9, two Javad TRE_G3T DELTA and a Septentrio POLARx4, at the campus of Curtin University in Perth, Australia. We connected four receivers (CUT0/1/2/3) to a single antenna (CUT00, Fig. S1a, online) and placed them in the roof-top plant room of building 402, suggesting that this group of receivers would be exposed to near-outdoor temperatures. For the rest of two receivers (SPA5/7) attached to another antenna (SPA00, Fig. S1b, online) mounted on the roof of building 407, we kept their hardware temperatures under control by situating them in a room with central air-conditioning system operating every day between 9:00 am and 21:30 pm. The distance between two antennas is about $358 \mathrm{~m}$.

Moreover, during the experiment we gathered the daily maximum temperatures recorded by a weather station located in Perth CBD (roughly $8 \mathrm{~km}$ away from our experimental sites) and managed by the Bureau of Meteorology, Australia. Additionally, we measured the internal hardware temperatures for three Trimble receivers (CUT0/2, SPA5) with a time resolution of about $1 \mathrm{~min}$ for a 10-day period (days 61-72 of 2015). The availability of temperature data would facilitate us to verify the BR-DCB temperature dependence. It is also worth mentioning that, we swapped the cables that link receivers CUT0/2 via the splitter with the antenna after day 56 of 2015, aiming to make it clear which part, among the antenna, the cable and the receiver hardware, is primarily responsible for BR-DCB temperature dependence.

In our BR-DCB retrieval and analysis, we will refer to five independent receiver-pairs that form four zero baselines and a short one. For each receiver-pair the characteristics of its multi-GNSS data set collected with a 30sec sampling interval are briefly summarized in Table 1 . We present, in the third column, the signal structures for different constellations, in terms of frequency band, tracking mode and modulation. For the data processing we set the cut-off elevation angle to 15 degrees so as to discard particularly noisy code measurements. We use the value of $30 \mathrm{~cm}$ for the $\sigma$ given in Eq. (2). We compute the satellite positions that are required for elevation angle determination using the broadcast ephemerides. In order to get the critical value $\chi_{\alpha}^{2}(t-1,0)$ for $T$, the significance level $\alpha$ is chosen equal to $5 \%$.

Due to the fact that we have generated a large set of BR-DCB results, we will restrict our following discussions to the most representative ones for simplicity's sake.

\subsection{Intra-day stability in BR-DCB time-wise estimates}


Before relying on $T$ as a measure for testing the intra-day stability of $\widehat{b}(i)$, one needs to first check whether the $\widehat{b}(i)$ on every experimental day are normally distributed or not. For this purpose, we depict the box plot as well as the histogram of $2880 \widehat{b}(i)$ samples retrieved for receiver-pair SPA5-CUT0 (Trimble-Trimble) and GPS constellation at day 336 of 2014 (an arbitrary choice) in Fig. 1a, b.

The box plot (Fig. 1a) splits the $\widehat{b}(i)$ samples into quartiles. The left and right edges of the central rectangle show the first $(\mathrm{Q} 1)$ and third $(\mathrm{Q} 3)$ quartiles, and the band inside the rectangle represents the second quartile (the median, Q2). The width of the rectangle (Q3-Q1) is also known as the inter-quartile range (IQR). Two horizontal lines, called whiskers, extend from the front and back of the rectangle. The end of the front (back) whisker takes the value Q1-1.5×IQR (Q3+1.5×IQR). The $\bar{b}(i)$ samples that are not included between the whiskers are depicted as outliers with black dots. Since the Q2 lies in the middle of the rectangle and the whisker lengths are the same, the distribution of the $\widehat{b}(i)$ samples is thereby symmetric. It is remarked that for a symmetric distribution the mean is approximately equal to the Q2.

Note, however, that confirming the symmetry with the use of box plot is essential for normality testing, but it yields only half the picture. We therefore need to take advantage of the histogram that is a good complement to the box plot and suits particularly well for displaying the shape of a distribution. When constructing the histogram (Fig. 1b), we first divide the entire range of $\widehat{b}(i)$ samples into a total of 40 equal-sized bins. Next we count the relative number of $\widehat{b}(i)$ samples that fall into each bin, and then represent it using a bar with height proportional to the count and width equal to the bin size (0.15 ns). Using this histogram as a backdrop, we further superimpose an empirical normal probability density function (PDF) curve on it, which has the same empirical mean (-1.80 ns) and standard deviation (0.97 ns) as the $\hat{b}(i)$ samples. Apparently, one can see from Fig. $1 \mathrm{~b}$ that the empirical normal curve agrees fairly well with the histogram. On the other hand, we 'predict' the values that five descriptive statistics underlying the box plot should take if the normality of $\widehat{b}(i)$ samples holds true. The 'predicted' values, shown as blue vertical lines in Fig. 1b, are in reasonable agreement with their empirical counterparts in Fig. 1a that are indicated by red vertical lines. These findings together suggest that the $\hat{b}(i)$ samples tested can indeed be assumed to follow a normal distribution.

Our discussion up to this point is general and applicable to all days’ $\hat{b}(i)$. Consequently, we are now allowed to compare the $T$ values computed using Eq. (3) against their critical values $\chi_{\alpha}^{2}(t-1,0)$ (Fig. 1c). We have to point out that, the $T$ as well as the $\chi_{\alpha}^{2}(t-1,0)$ values tend to decrease remarkably at some experimental days with fewer usable epochs $(t)$. From Fig. 1c it follows that $T<\chi_{\alpha}^{2}(t-1,0)$ always holds, which can be taken as an indication that $\widehat{b}(i)$ samples do not show any statistically significant intra-day changes. Motivated by this fact we decide to base our following analyses on the DWA estimates, denoted as $\bar{b}$ in Eq. (3). 
Table 2 summarizes two descriptive statistics (empirical mean and standard deviation) of $\bar{b}$ for different GNSS constellations and all receiver-pairs involved in this study. An in-depth investigation into the numerical values presented therein raises a number of interesting findings. First of all, the BR-DCB mean value depends on at least three factors, namely receiver-pair, GNSS constellation and frequency-pair. We see, for example, that the offset between QZSS (L1-L5) and Galileo (E1-E5a) BR-DCB mean values is still greater than 15 ns, even though they refer to a common receiver-pair CUT0-CUT1 (Trimble-Septentrio) and an overlapping frequency-pair. This situation becomes even more complicated for the BeiDou BR-DCB results, in which we additionally find their possible satellite type dependence. Furthermore, we may acquire some information about the BR-DCB variations during the experimental period from the standard deviation values. For all receiver-pairs other than the CUT0CUT3 (Trimble-Javad), their BR-DCB standard deviation values across different GNSS constellations range in size from $0.61 \mathrm{~ns}$ to $0.16 \mathrm{~ns}$. This implies very moderate long-term temporal variability in these BR-DCB estimates. The unexpectedly high BR-DCB standard deviations for CUT0-CUT3 are probably caused by BR-DCB estimate anomalies, especially for GPS, Galileo and QZSS constellations. With respect to a given receiver-pair, the BeiDou BR-DCB standard deviation values always manifest much less satellite type dependence than the mean values. We thereby surmise that the three sets of BeiDou BR-DCB estimates corresponding to distinct satellite types might exhibit similar temporal variations. In the following discussion, we will revisit in more detail some of these issues in an attempt to clarify the possible reasons behind them.

\subsection{Satellite-type Dependent BeiDou BR-DCBs}

Concerning the first three receiver-pairs involved in Table 1, we show for each of them three time series of BeiDou BR-DCBs retrieved respectively from GEO/IGSO/MEO satellites’ measurements (Fig. 2). The inconsistency between GEO/IGSO/MEO time series is quite apparent for two receiver-pairs of mixed types (Fig. 2a, c). This is largely caused by the code ISTBs to which both receiver-pairs' BeiDou measurements are subjected. One possible explanation for code ISTB occurrence might be the multipath effects that do not average out in the same manner for the different ground tracks of GEO/IGSO/MEO satellites. For the special case when two BeiDou-capable receivers of the same type are considered, the almost identical sensitivity of them to the multipath effects would very likely yield quite small or even absent code ISTBs. This is the reason why the three time series obtained for receiver-pair CUT0-CUT2 (Trimble-Trimble) overlap one another fairly well (Fig. 2b).

For each receiver-pair we also provide a revealing summary of the offsets between any two of GEO/IGSO/MEO time series with parallel box plots (Fig. S2, online). Here we focus first on the analysis of the three plots depicted for CUT0-CUT3 (Trimble-Javad). Both the GEO-IGSO and the GEO-MEO plots have nearly equal medians $(0.4 \mathrm{~ns})$ and are skewed to the left. However, the latter plot has greater range (about $1.6 \mathrm{~ns})$ than the former one (about $1.1 \mathrm{~ns}$ ), thus implying much larger variability. Moreover, most offsets between GEO and nonGEO time series would be concentrated on the right of the mean, with extreme values to the left. Conversely, the IGSO-MEO plot is reasonably symmetric and has median very close to $0 \mathrm{~ns}$. This plot also has a much smaller range (0.8 ns) than the other two, which suggests the least variability in the offsets between two non-GEO time series. As to CUT0-CUT2 (Trimble-Trimble) all the plots are symmetric, with medians around 0 ns and ranges below 0.2 ns. This again confirms that the GEO/IGSO/MEO time series for this receiver-pair are virtually 
consistent. The situation for CUT0-CUT1 (Trimble-Septentrio) is somewhat in between: none of the plots shows prominent skewness; any two of GEO/IGSO/MEO time series would have essential differences as the medians of three plots vary from $0.2 \mathrm{~ns}$ to $0.4 \mathrm{~ns}$; the variability in different groups of offsets seems to be comparable and moderate since the ranges stay around $0.5 \mathrm{~ns}$ for all three plots.

Returning to Fig. 2, we do find that for each receiver-pair the GEO/IGSO/MEO time series share high similarity in their temporal variations despite the possible presence of code ISTB induced offsets. More interestingly, all the time series obtained for the first and the third receiver-pairs (Fig. 2a, c) underwent concurrent abrupt changes after upgrading the firmware version of CUT0 (Trimble) on day 175 of 2013. The fundamental reason for this is that the digital signal processing inside the receiver, on which the receiver's DCB would be partially dependent, may alter as soon as the firmware upgrade has been done. Since the other Trimble receiver CUT2 was also upgraded to the same firmware version as the CUT0 at that day, the effect of firmware upgrade on each receiver's DCB is almost identical and thus cancels out in their BR-DCB estimates (Fig. 2b). Likewise, the BR-DCB estimates for receiver-pair CUT0-CUT3 and constellations Galileo, GPS and QZSS (L1-L5) all exhibit a significant increase as a response to firmware upgrade of CUT3 (Javad) on day 261 of 2013 (Fig. S3, online). This fact thereby explains why these three groups of BR-DCB estimates have much higher standard deviations than the remaining groups (Table 2). Regarding the cases so far investigated the BR-DCB changes caused by firmware upgrade can vary in size from 0.4 ns (Fig. 2a) to 3 ns (Fig. S3a, online).

Additionally, all the time series resemble each other very closely in how they change over time irrespective of receiver-pair. This suggests that the long-term changes in ambient temperature around the four receivers involved are a major cause. To help verify this issue we will now turn to a detailed analysis of more experimental results.

\subsection{Temperature-induced BR-DCB Variations}

As starting point we investigate the time series of both the daily temperatures, as well as the BR-DCB estimates for receiver-pair CUT2-CUT0 and BeiDou IGSO covering the year 2013 (Fig. 3a). Obviously, the two time series look very much alike, thus suggesting quite a striking positive correlation. We measure the linear dependence between both time series in terms of the Pearson Correlation Coefficient (PCC). By definition, PCC is the covariance of the two time series divided by the product of their standard deviations [35]. In a two-tailed test with significance level set at $5 \%$, the absolute value of PCC greater than 0.182 would indicate a statistically significant dependence for a sample size larger than 200. This fact indeed holds in our case as the value computed for the PCC is about 0.79. Another example concerns the 1-year (2014) time series of BR-DCB estimates for receiver-pair SAP5-CUT0 and constellation GPS, along with that of daily temperatures (Fig. 3b). The dependence between the two time series, from a statistical point of view, is even more significant than the former case since the PCC value now equals approximately 0.89 .

We also graphically display the same data as a scatter plot that has a collection of points (Fig. S4, online). Each point has the value of daily temperature (explanatory variable) determining the position on the horizontal axis, and the value of the BR-DCB estimate (response variable) determining the position on the vertical axis. The scatter plot gives a good visual picture of the relationship between the two variables. One can, for instance, easily identify 
that the points of each subplot follow a strong linear pattern. Overlaid on the scatter plot is the line of best fit in red that is found by minimizing the sum of the squares of the distances of the points to the line. The straight line so obtained can well represent the trend in the BR-DCB estimate versus daily temperature data and its slope reflects the rate of BR-DCB change that is shown to be on the order of 27 to 94 ps per degree Celsius. Perhaps more interestingly, after linearly detrending the BR-DCB time series with respect to the temperature, a further examination of the residual BR-DCB estimates for dependence on other factors becomes plausible and well deserves future analysis.

Next, we seek to gain a better understanding of BR-DCB estimate dependence on temperature. For this purpose we manually swapped the cables linking two Trimble receivers CUT0 and CUT2 via the splitter with one shared antenna, which took place on February 25, 2015 (DOY 56) at 10:00 am local time (UTC+8). After 20 days on March 17, 2015 (DOY 76) at 15:00 pm, we once again swapped both cables so as to bring the original receiverantenna connectivity back. Two daily time series of BR-DCB time-wise estimates for this receiver-pair and BeiDou GEO at DOYs 56 and 76 contain a total of two abrupt changes, which show up immediately after the cables are swapped and appear to be equal in absolute magnitude, but opposite in sign (Fig. 4). Hence, it follows that the overall size of BR-DCB estimates is not only dependent on the receivers involved exclusively, but rather on the cables and of course, the antenna(s).

At the same time, we, however, conclude that the BR-DCB temperature dependence is primarily due to receiver hardware. We employ first of all the BR-DCB versus daily temperature data spanning days 1-75 of 2015 to confirm this conclusion. The BR-DCB estimates shown here refer to the same receiver-pair and constellation as discussed above. For the convenience of the later discussion, we split the data into two groups according to whether they were produced before or after the first cable swapping (day 56) and then display them separately in Fig. 5a, b. Importantly, we can readily see that a high positive correlation between daily temperatures and BR-DCB estimates always exists and is found to be insensitive to cable swapping. We take this as an indication that the cables are unlikely to be responsible for BR-DCB temperature dependence, otherwise a negative correlation between the two sets of data in Fig. 5b would have been recognizable.

Nevertheless, at this point we are still unable to distinguish the receiver hardware from the antenna as a standalone factor inducing dependence of BR-DCB estimates upon temperature. This is because, same as the antenna, both receivers CUT0 and CUT2 are also subjected to ambient (outdoor) temperatures. Remarkably, the hardware temperatures observed for receiver CUT0 over a period as long as one day could be closely associated with the local time (Fig. 5c). They rise gradually during the daytime hours and begin to fall at about 19:00 pm since the Sun is setting. Additionally, the daily maximum temperatures measured on DOYs 62 and 71 differ by approximately 7 degrees Celsius (Fig. 5b) and this, in turn, has led to a profound increase of up to 5 degrees Celsius in hardware temperatures inside CUT0 over these two days. The situation for the other receiver CUT2 is largely the same, but it always experiences lower hardware temperatures than CUT0 although they are from the same manufacturer and have identical design. This implies that the differences between the hardware temperatures of both receivers would in principle present and thus very possibly manifest themselves as a general cause of BRDCB variations. 
From Fig. S5a (online), which shows a statistically insignificant correlation (PCC=0.01) between BR-DCB estimates for receiver-pair SPA5-SPA7 (Trimble-Javad) and the daily temperatures covering a period of up to 1 year, we would eventually be able to confirm that the receiver hardware remains the sole factor accounting for BRDCB temperature dependence. This can be understood from two different, but complementary, points of view. As mentioned earlier, both receivers involved have been placed inside a controlled-temperature room. Thus, taking as an example the receiver SPA5 (Fig. S5b, online), we see much better within-day stability and more promising between-day repeatability in its hardware temperatures measured on DOYs 62 and 71 as compared to CUT0/CUT2 (Fig. 5c). In particular, the daily temperature data oscillate only about 0.5 degrees Celsius for the time interval when the air-conditioning system is turned on (between 9:00 am and 21:30 pm). On the other hand, continuous exposure of the antenna shared commonly by both receivers to outdoor temperatures is still the case here, but the very striking statistical dependence between BR-DCB estimates and daily temperatures (see Fig. 3) does not show up any longer.

\section{Summary and Conclusions}

We introduced a simple method enabling time-wise BR-DCB retrieval with significant effectiveness. The method is simple because it takes advantage of dual-frequency, code-only measurements from a pair of co-located receivers. This method is also effective since it does not require any externally provided information and is applicable even to QZSS that has, so far, launched only a single satellite. Due to its reliance upon zero or short baseline set up, this method might be more demanding than customary ones (see [23] and references therein), but on the other hand it retrieves BR-DCB estimates free of ionospheric leveling and/or modeling errors, thus serving our study best. When applying such a method to multi-GNSS data with a standard 30 second sampling rate collected by six continuously operating receivers from three manufactures, we get a large set of BR-DCB time-wise estimates that correspond to different receiver-pairs, frequency-pairs and constellations. Notably, for each pair of receivers the BeiDou code measurements from GEO, IGSO and MEO satellites are treated as if they were from three different constellations. In doing so, we have been able to obtain three sets of BeiDou BR-DCB estimates for one receiver-pair and then check their consistency.

By means of statistical hypothesis testing, the daily BR-DCB time-wise estimates are found to be normally distributed and sufficiently stable, thus lending themselves to being represented by one DWA estimate. The BeiDou BR-DCB DWA estimates derived, respectively, from GEO, IGSO and MEO satellites' measurements indeed show inconsistency. This occurs generally for the receiver-pairs from different manufactures and originates mainly from code ISTBs that differ at two frequencies. In our case, the offsets between GEO and non-GEO derived BeiDou BR-DCB estimates spanning a 2-year period could have a median of 0.4 ns and a range of $1.6 \mathrm{~ns}$. The overall size of BR-DCB estimates may exhibit an abrupt change once the receiver firmware version has been upgraded. The long-term temporal variability in BR-DCB estimates seems to be caused by receiver hardware temperature variations. The very striking statistical correlation between BR-DCB estimates and daily maximum ambient temperatures begins to vanish provided that we place both receivers involved in a controlled-temperature room. 
Acknowledgements We would like to express our gratitude to Dr. Oliver Montenbruck and Dr. Jean-Marie Sleewaegen for their thoughtful suggestions and extensive discussions. Special thanks go to Dr. Nandakumaran Nadarajah and Mr. Matt Carver for collecting the multi-GNSS experimental data, and to Bureau of Meteorology (Australia) for providing the online climate data. This work has been executed in the framework of the Positioning Program Project 1.19 “Multi-GNSS PPP-RTK Network Processing” of the Cooperative Research Centre for Spatial Information (CRC-SI). This work was also partially funded by the Chinese Academy of Sciences (CAS) and the Royal Netherlands Academy of Arts and Sciences (KNAW) joint research project 'Compass, Galileo and GPS for improved ionosphere modelling'. The second author is the recipient of an Australian Research Council (ARC) Federation Fellowship (project number FF0883188). All this support is gratefully acknowledged.

Conflict of interest The authors declare that they have no conflict of interest.

\section{References}

1. Li Z, Yuan Y, Wang N et al (2015) SHPTS: towards a new method for generating precise global ionospheric TEC map based on spherical harmonic and generalized trigonometric series functions. J Geodesy 89: 331-345

2. Liu L, Wan W, Chen Y et al (2011) Solar activity effects of the ionosphere: A brief review. Chin Sci Bull 56: 1202-1211

3. Mannucci A, Wilson B, Yuan D et al (1998) Global mapping technique for GPS-derived ionospheric total electron content measurements. Radio Sci 33: 565-582

4. Komjathy A, Wilson B, Pi X et al (2010) JPL/USC GAIM: On the impact of using COSMIC and ground-based GPS measurements to estimate ionospheric parameters. J Geophys Res 115: A02307

5. Hernández-Pajares M, Juan J, Sanz J et al (2009) The IGS VTEC maps: a reliable source of ionospheric information since 1998. J Geodesy 83: 263-275

6. Gu S, Shi C, Lou Y et al (2015) Ionospheric effects in uncalibrated phase delay estimation and ambiguityfixed PPP based on raw observable model. J Geodesy 89: 447-457

7. Chen G, Zhao Q (2014) Near-field surface displacement and permanent deformation induced by the Alaska Mw 7.5 earthquake determined by high-rate real-time ambiguity-fixed PPP solutions. Chin Sci Bull 59: 47814789

8. Hernández-Pajares M, Juan J M, Sanz J et al (2011) The ionosphere: effects, GPS modeling and the benefits for space geodetic techniques. J Geodesy 85: 887-907

9. Liu Z, Skone S, Gao Y et al (2005) Ionospheric modeling using GPS data. GPS Solut 9: 63-66

10. Yuan Y, Tscherning C C, Knudsen P et al (2008) The ionospheric eclipse factor method (IEFM) and its application to determining the ionospheric delay for GPS. J Geodesy 82: 1-8

11. Yuan Y, Ou J (2002) Differential areas for differential stations (DADS): A new method of establishing grid ionospheric model. Chin Sci Bull 47: 1033-1036

12. Spits J, Warnant R (2008) Total electron content monitoring using triple frequency GNSS data: A three-step approach. J Atmos Sol-Terr Phy 70: 1885-1893

13. Le A Q (2006) Impact of Galileo on global ionosphere map estimation. J Navigation 59: 281-292

14. Tang W, Jin L, Xu K (2014) Performance Analysis of Ionosphere Monitoring with BeiDou CORS Observational Data. J Navigation 67: 511-522

15. Yang Y, Li J, Xu J et al (2011) Contribution of the compass satellite navigation system to global PNT users. Chin Sci Bull 56: 2813-2819

16. Lou Y, Liu Y, Shi C et al (2014) Precise orbit determination of BeiDou constellation based on BETS and MGEX network. Sci Rep. doi:10.1038/srep04692

17. Steigenberger P, Hugentobler U, Montenbruck O et al (2011) Precise orbit determination of GIOVE-B based on the CONGO network. J Geodesy 85: 357-365

18. Hauschild A, Steigenberger P, Rodriguez-Solano C (2012) Signal, orbit and attitude analysis of Japan's first QZSS satellite Michibiki. GPS Solut 16: 127-133 
19. Kawano I, Mokuno M, Kogure S et al (2004) Japanese experimental GPS augmentation using quasi-zenith satellite system (QZSS). In: Proceedings of the Institute of Navigation (ION) GNSS 2004, Long Beach, 2004

20. Sardon E, Rius A, Zarraoa N (1994) Estimation of the transmitter and receiver differential biases and the ionospheric total electron content from Global Positioning System observations. Radio Sci 29: 577-586

21. Li Z, Yuan Y, Fan L et al (2014) Determination of the differential code bias for current BDS satellites. IEEE Trans Geosci Remote Sens 52: 3968-3979

22. Sardón E, Zarraoa N (1997) Estimation of total electron content using GPS data: How stable are the differential satellite and receiver instrumental biases? Radio Sci 32: 1899-1910

23. Li Z, Yuan Y, Li H et al (2012) Two-step method for the determination of the differential code biases of COMPASS satellites. J Geodesy 86: 1059-1076

24. Montenbruck O, Hauschild A, Steigenberger P (2014) Differential code bias estimation using Multi-GNSS observations and global ionosphere maps. Navig J Inst Navig 61: 191-201

25. Ciraolo L, Azpilicueta F, Brunini C et al (2007) Calibration errors on experimental slant total electron content (TEC) determined with GPS. J Geodesy 81: 111-120

26. Brunini C, Azpilicueta F J (2009) Accuracy assessment of the GPS-based slant total electron content. J Geodesy 83: 773-785

27. Coster A, Williams J, Weatherwax A et al (2013) Accuracy of GPS total electron content: GPS receiver bias temperature dependence. Radio Sci 48: 190-196

28. Zhang D, Shi H, Jin Y et al (2014) The variation of the estimated GPS instrumental bias and its possible connection with ionospheric variability. Sci China Techn Sci 57: 67-79

29. Zhang D, Zhang W, Li Q et al (2010) Accuracy analysis of the GPS instrumental bias estimated from observations in middle and low latitudes. Ann Geophys 28: 1571-1580

30. Zhang W, Zhang D, Xiao Z (2009) The influence of geomagnetic storms on the estimation of GPS instrumental biases. Ann Geophys 27: 1613-1623

31. Zhong J, Lei J, Dou X et al (2015) Is the long-term variation of the estimated GPS differential code biases associated with ionospheric variability? GPS Solut. doi: 10.1007/s10291-015-0437-5

32. Zhang B, Ou J, Yuan Y et al (2012) Extraction of line-of-sight ionospheric observables from GPS data using precise point positioning. Sci China Earth Sci 55: 1919-1928

33. Stephens P, Komjathy A, Wilson B et al (2011) New leveling and bias estimation algorithms for processing COSMIC/FORMOSAT-3 data for slant total electron content measurements. Radio Sci. doi: 10.1029/2010RS004588

34. Nadarajah N, Teunissen P, Raziq N (2013) BeiDou Inter-Satellite-Type Bias Evaluation and Calibration for Mixed Receiver Attitude Determination. Sensors 13: 9435-9463

35. Freedman D A (2009) Statistical models: theory and practice. Cambridge University Press, London 
Table 1 A general overview of the characteristics of five experimental receiver-pairs

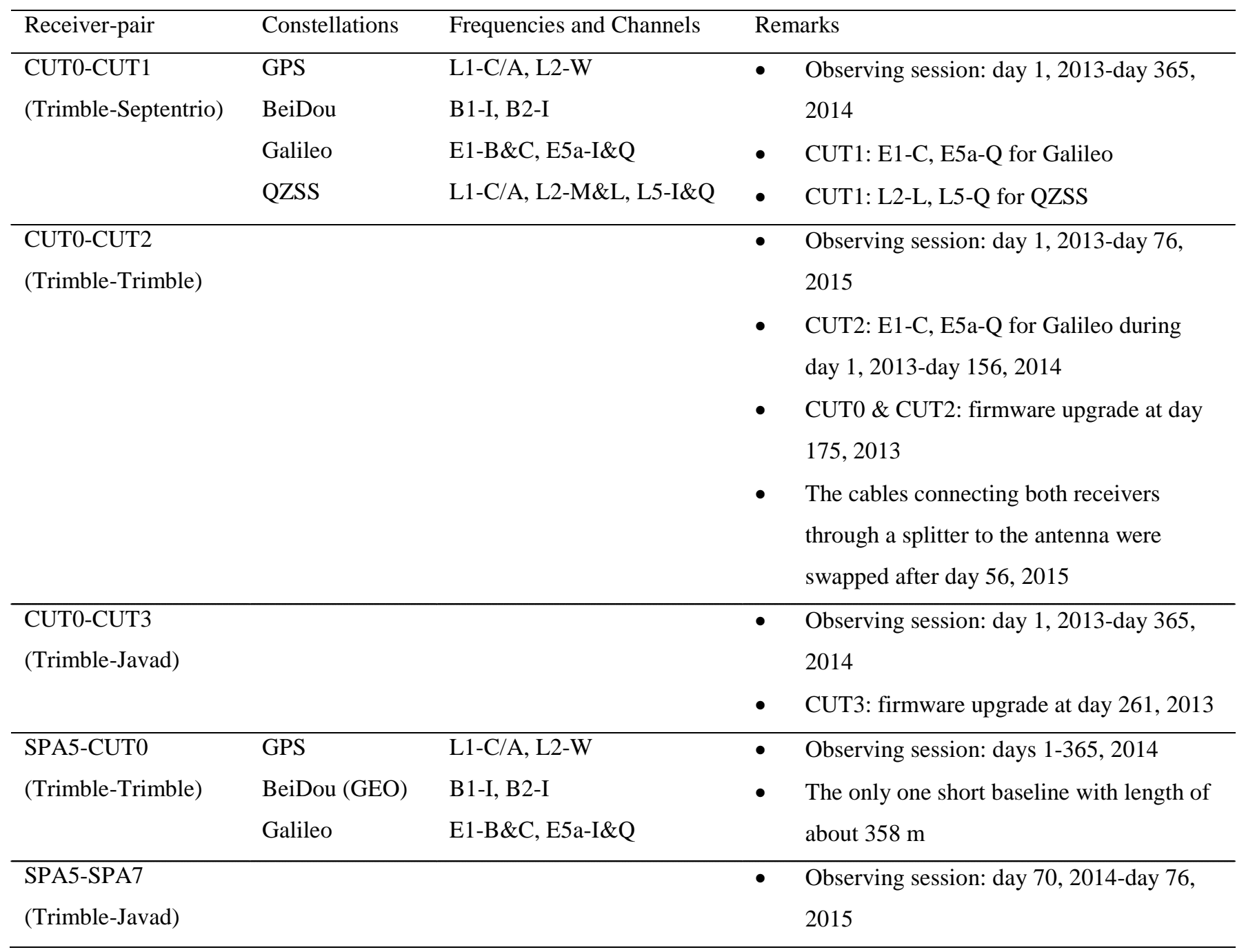

\section{Table 2}

Descriptive statistics for all sets of BR-DCB DWA estimates: empirical mean (ns) | standard deviation (ns).

For the two receiver-pairs highlighted in bold italic font, the calculation of statistics did not involve the BR-DCB DWA estimates computed for the first 76 days of 2015.

\begin{tabular}{lccccccc}
\hline Receiver-pair & GPS & \multicolumn{3}{c}{ BeiDou } & \multirow{2}{*}{ Galileo } & \multicolumn{2}{c}{ QZSS } \\
\cline { 3 - 5 } & & GEO & IGSO & MEO & & L1-L2 & L1-L5 \\
\hline CUT0-CUT1 & $-13.52 \mid 0.33$ & $5.60 \mid 0.37$ & $5.42 \mid 0.32$ & $5.24 \mid 0.33$ & $0.30 \mid 0.16$ & $-12.89 \mid 0.30$ & $-15.19 \mid 0.17$ \\
\hline CUT0-CUT2 & $0.57 \mid 0.22$ & $-0.62 \mid 0.20$ & $-0.64 \mid 0.19$ & $-0.58 \mid 0.22$ & $0.36 \mid 0.16$ & $0.36 \mid 0.35$ & $0.50 \mid 0.21$ \\
\hline CUT0-CUT3 & $-12.38 \mid 0.94$ & $-95.13 \mid 0.44$ & $-95.46 \mid 0.38$ & $-95.44 \mid 0.42$ & $-11.65 \mid 1.51$ & $-12.17 \mid 1.48$ & $-26.92 \mid 1.08$ \\
\hline SPA5-CUT0 & $-2.11 \mid 0.61$ & $-2.52 \mid 0.47$ & n/a & n/a & $2.57 \mid 0.28$ & n/a & n/a \\
\hline SPA5-SPA7 & $-12.77 \mid 0.20$ & $-93.20 \mid 0.22$ & n/a & n/a & $-11.01 \mid 0.16$ & n/a & n/a \\
\hline
\end{tabular}


Fig. 1 The box plot (subplot a) and histogram (subplot b) of BR-DCB time-wise estimates for receiver-pair SPA5-CUT0 (Trimble-Trimble) and constellation GPS on day 336 of 2014: the normal PDF curve with mean -1.80 ns and standard deviation $0.97 \mathrm{~ns}$ is overlaid on the histogram; the five vertical lines in blue indicate the empirical values of five descriptive statistics associated with the box plot. Results of statistical hypothesis testing for checking the intra-day stability in daily BRDCB time-wise estimates during year 2014 (subplot c): test statistics (blue dots) versus critical values (red dots).

Fig. 2 Time series of BeiDou DWA estimates derived, respectively, from GEO (in black), IGSO (in red) and MEO (in green) satellites’ measurements for three receiver-pairs: CUT0-CUT1 (Trimble-Septentrio, subplot a), CUT0-CUT2 (Trimble-Trimble, subplot b) and CUT0-CUT3 (Trimble-Javad, subplot c). The two arrows in blue specify day 175 of 2013 when two Trimble receivers CUT0 and CUT2 commonly underwent a firmware upgrade.

Fig. 3 BR-DCB DWA estimates (blue dotted line) versus daily maximum ambient temperatures (red dotted line) as a function of DOY: results for receiver-pair CUT2-CUT0, constellation BeiDou (IGSO) and year 2013 have a PCC of 0.79 (subplot a); results for receiver-pair SPA5-CUT0, constellation GPS and year 2014 have a PCC of 0.89 (subplot b).

Fig. 4 The daily time series of BR-DCB time-wise estimates on DOYs 56 and 76 (2015) for receiver-pair CUT2-CUT0 and GPS constellation. The two instants when the cable swapping occurred are marked with dash lines.

Fig. 5 Before (subplot a) and after (subplot b) swapping the cables at day 56 of 2015, the BR-DCB DWA estimates for receiver-pair CUT2-CUT0 and constellation BeiDou (GEO) (blue dotted line) versus daily maximum ambient temperatures (red dotted line); The hardware temperatures with a 1-min time resolution for receivers CUT0 in blue and CUT2 in red versus local time (subplot c). 

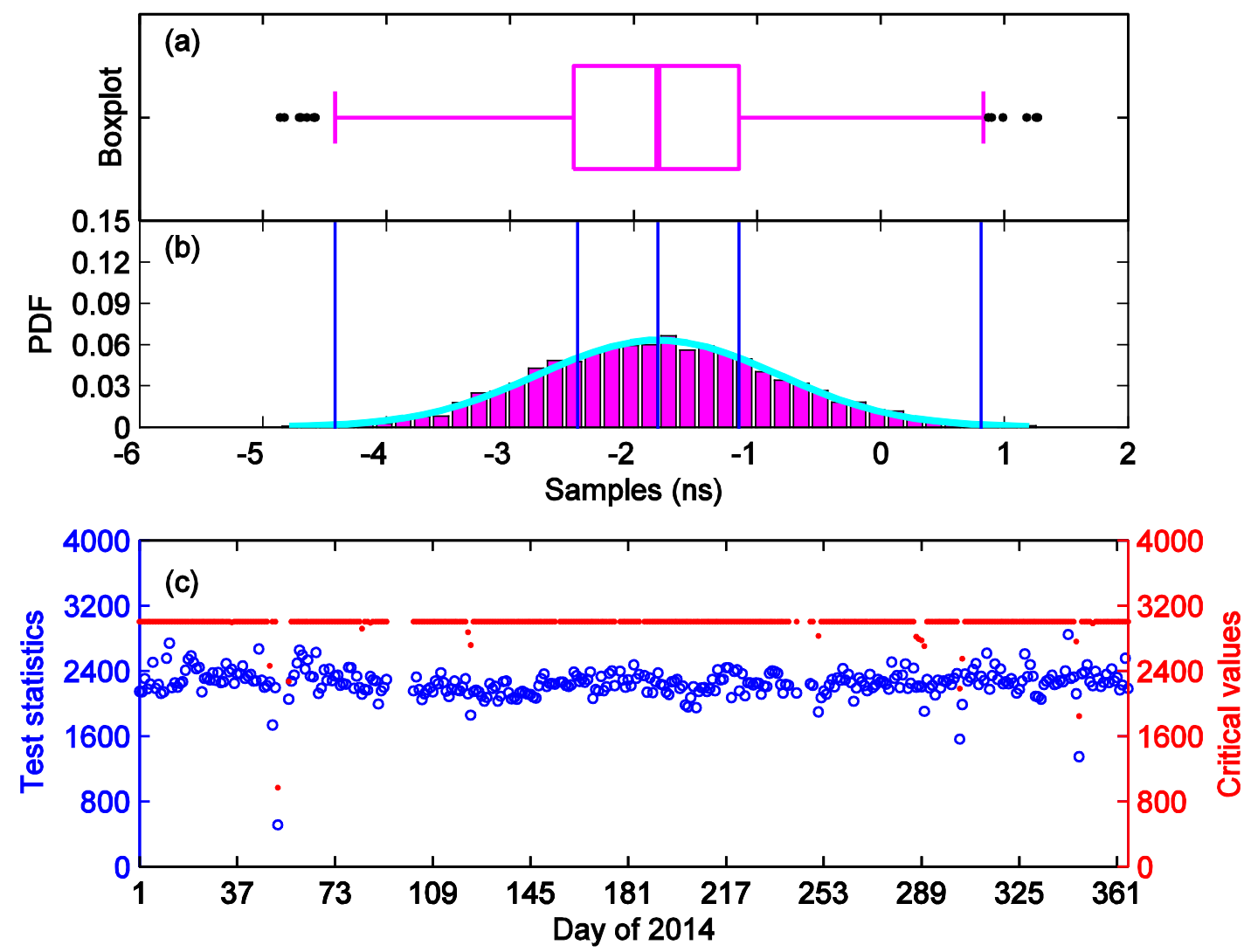


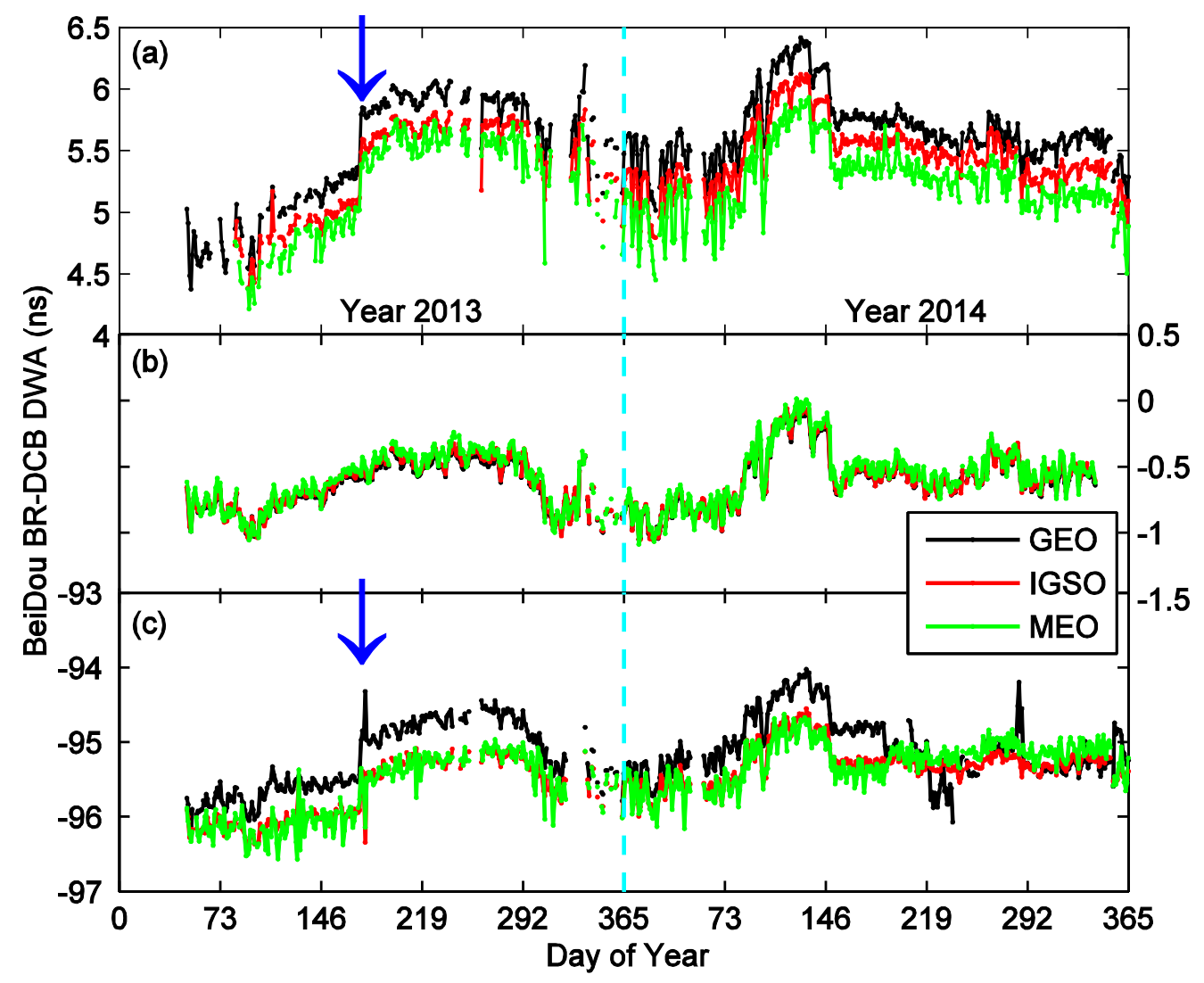




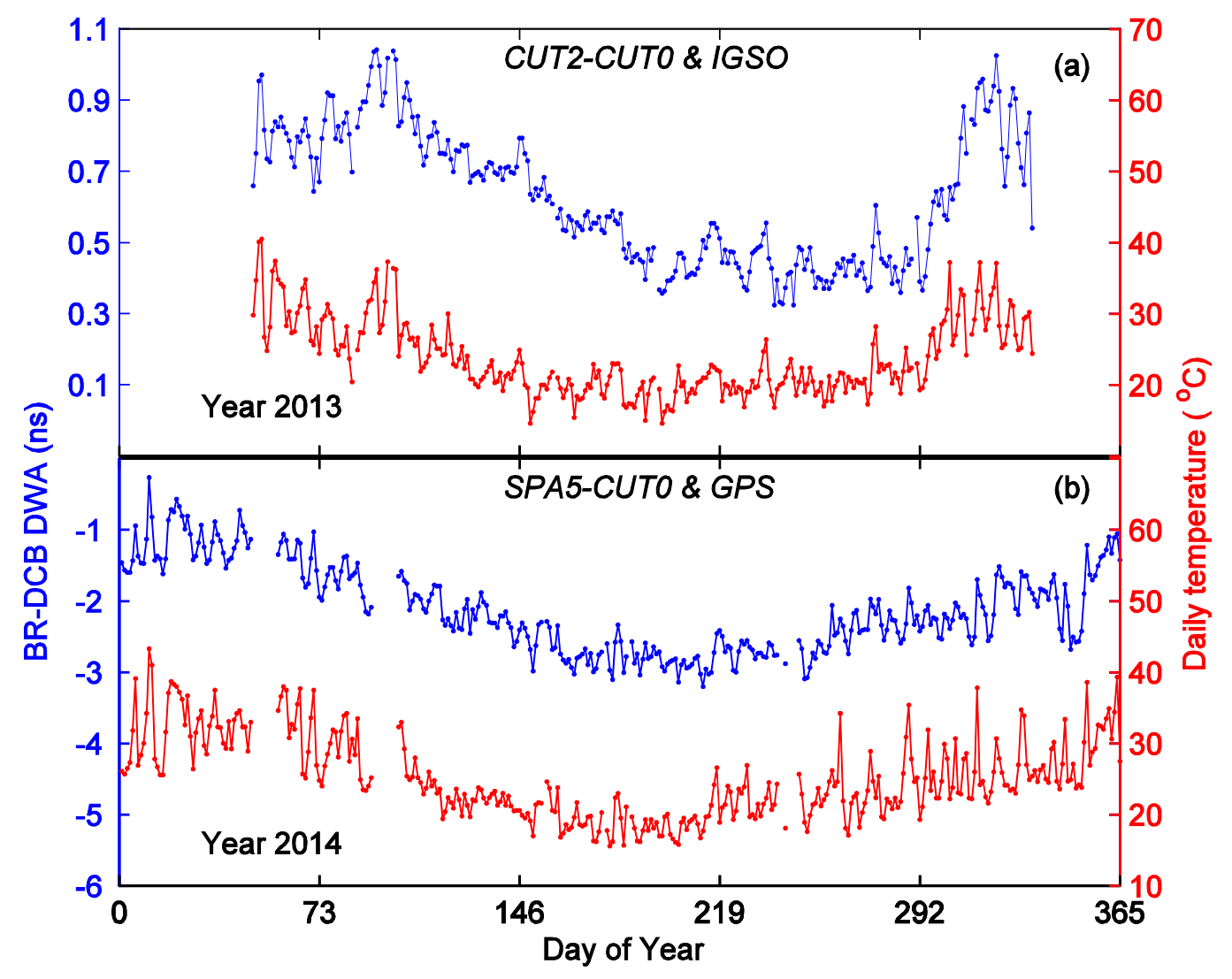




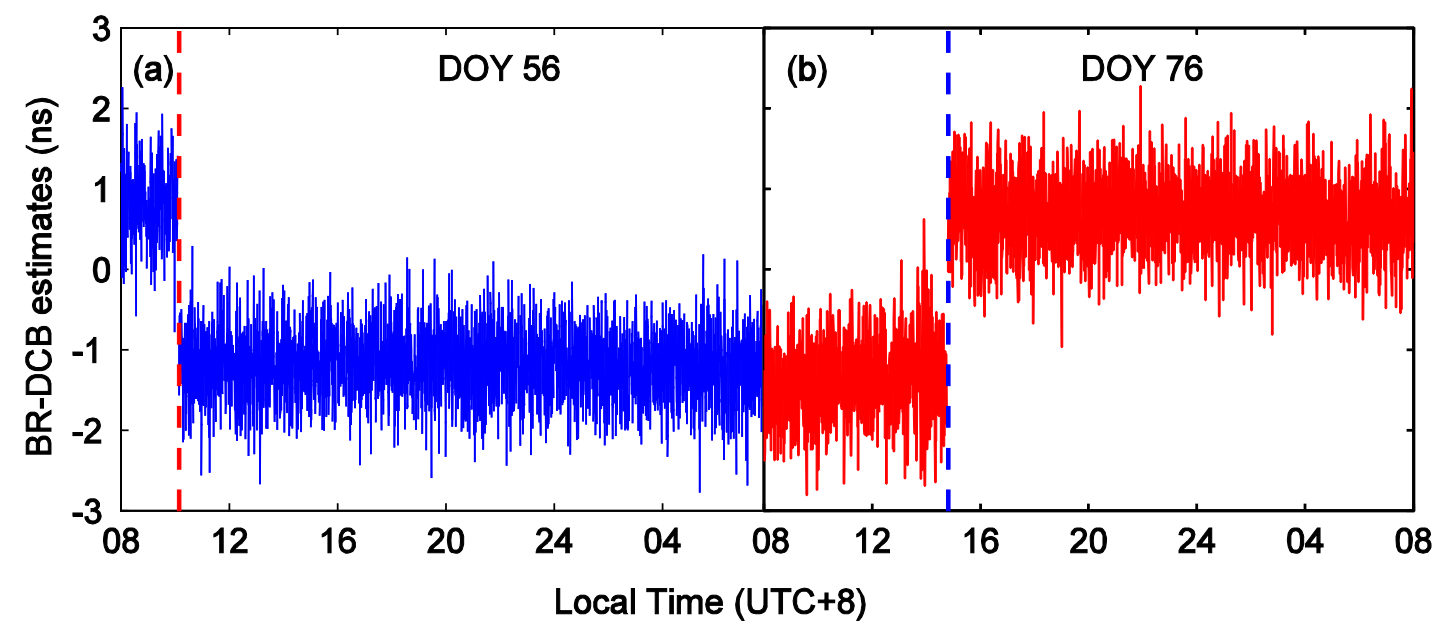



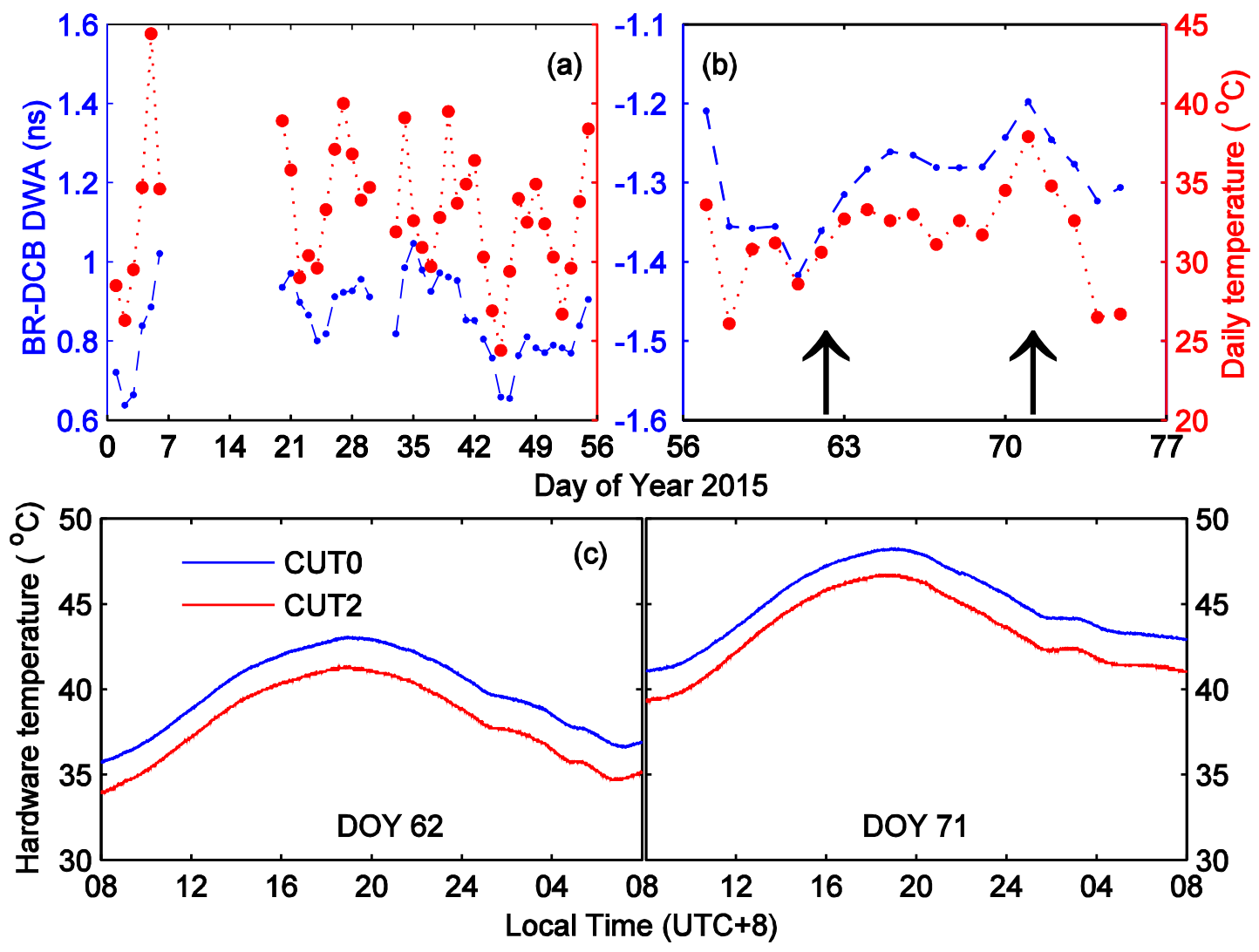


\section{Science Bulletin}

${ }^{\text {'6 }}$ Characterization of Multi-GNSS Between-Receiver Differential Code Biases Using Zero and Short Baselines"

Baocheng Zhang $^{1 *}$ and Peter J.G. Teunissen ${ }^{1,2}$

${ }^{1}$ GNSS Research Centre, Curtin University, Australia

${ }^{2}$ Geoscience and Remote Sensing, Delft University of Technology, The Netherlands

*Corresponding author: b.zhang@curtin.edu.au; Tel: 61-8-9266-7566; Fax: 61-8-9266-2703

\section{Supplementary Materials}



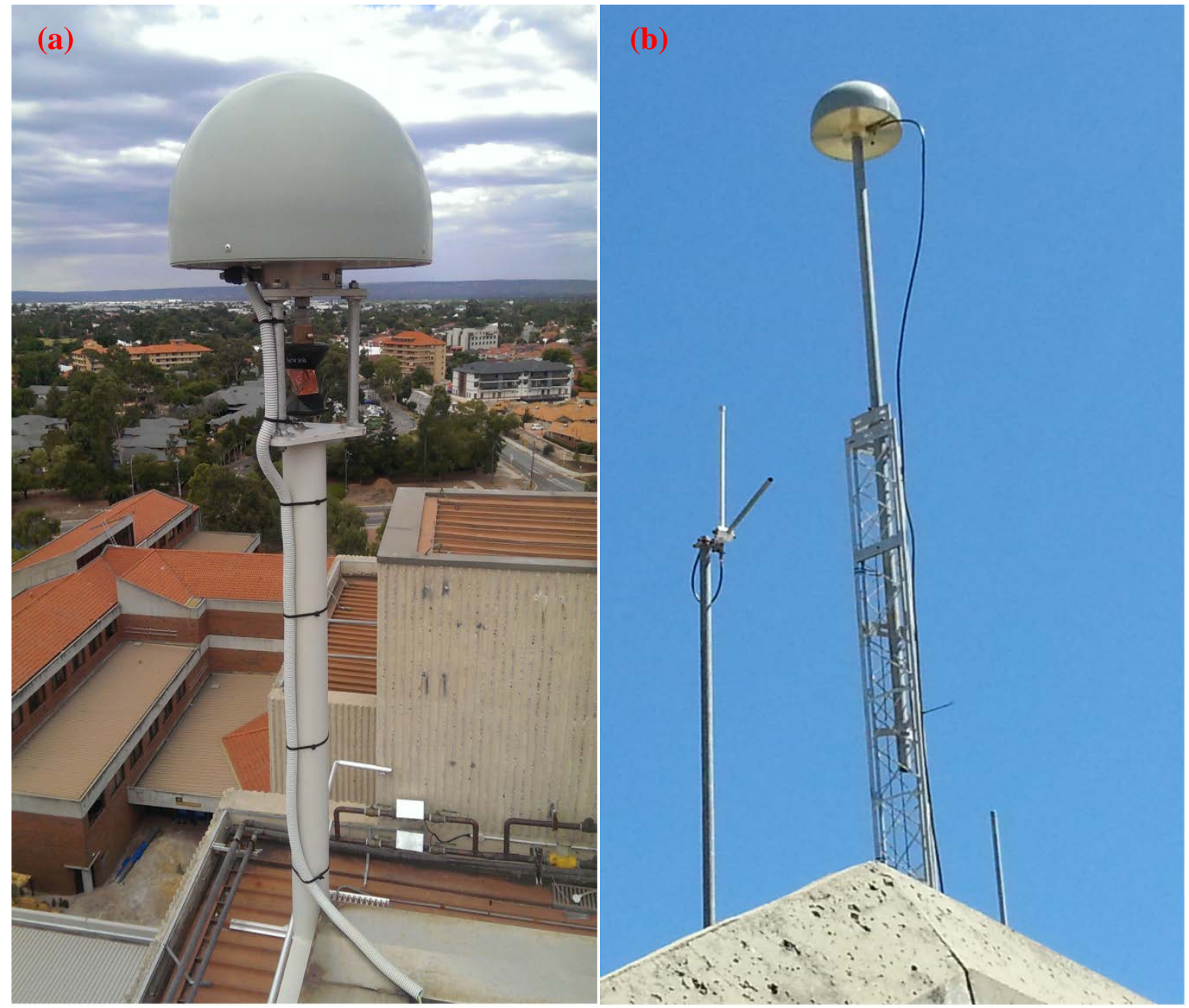

Fig. S1 Setup of CUT00 (subplot a) and SPA00 (subplot b): the distance between two antennas is about $358 \mathrm{~m}$. 


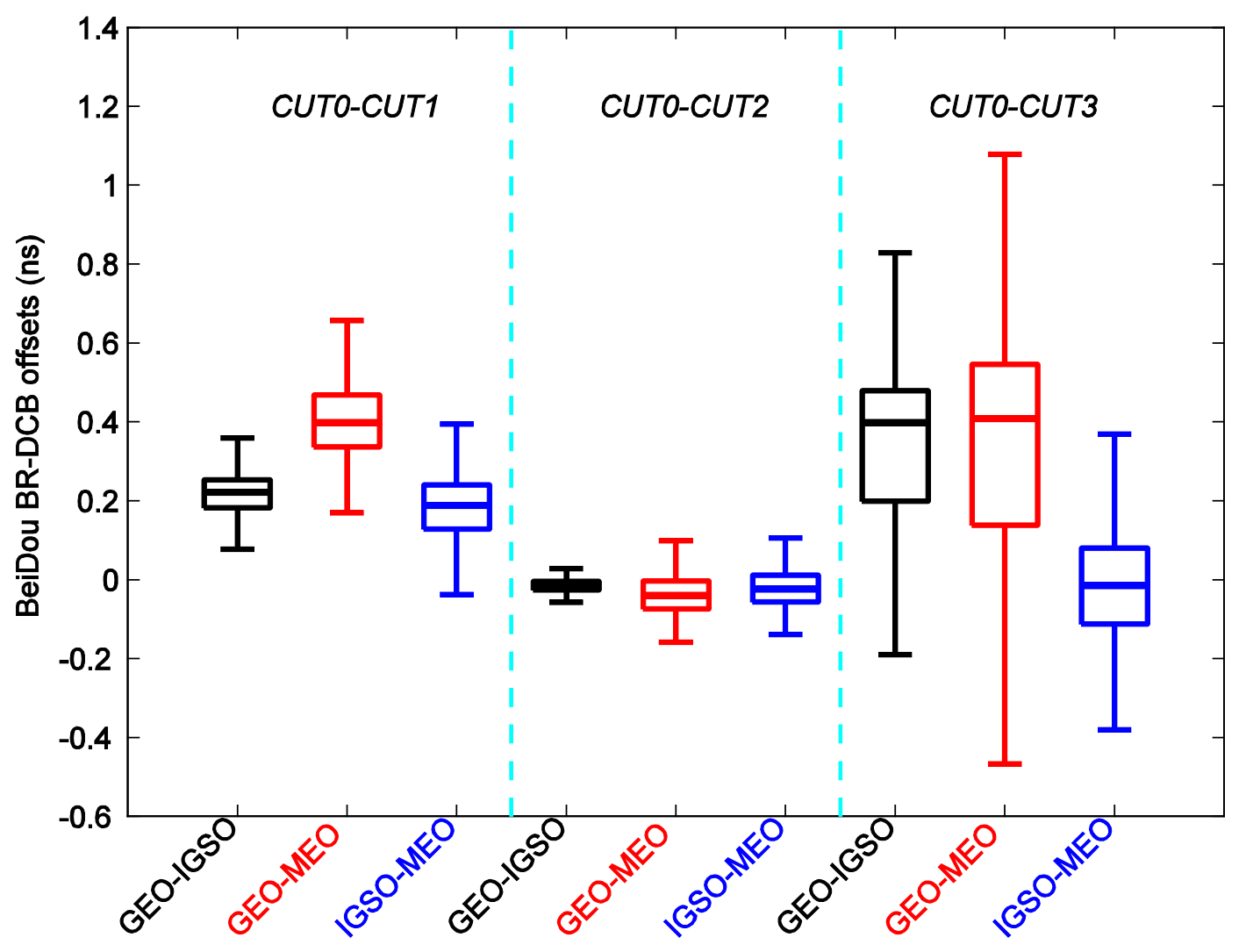

Fig. S2 Parallel box plots summarizing for each receiver-pair the offsets between any two of GEO, IGSO and MEO time series depicted in Fig. 2. 


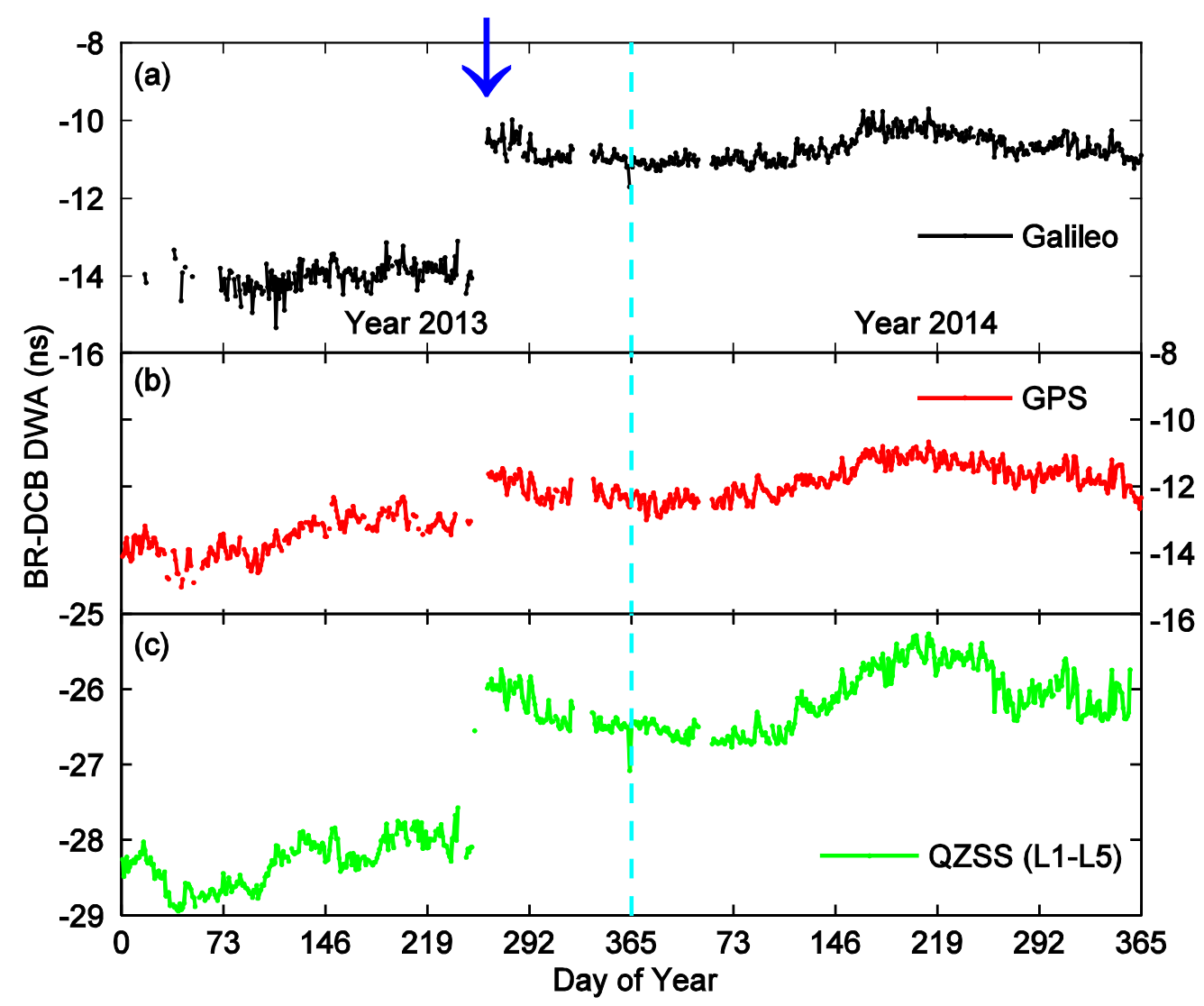

Fig. S3 Time series of BR-DCB DWA estimates for receiver-pair CUT0-CUT3 (Trimble-Javad) and constellations Galileo (in black, subplot a), GPS (in red, subplot b) and QZSS (frequency-pair L1-L5, in green, subplot c). The time period spans years 2013 and 2014. The arrow in blue indicates day 261 of 2013 when the firmware version of CUT3 was upgraded. 


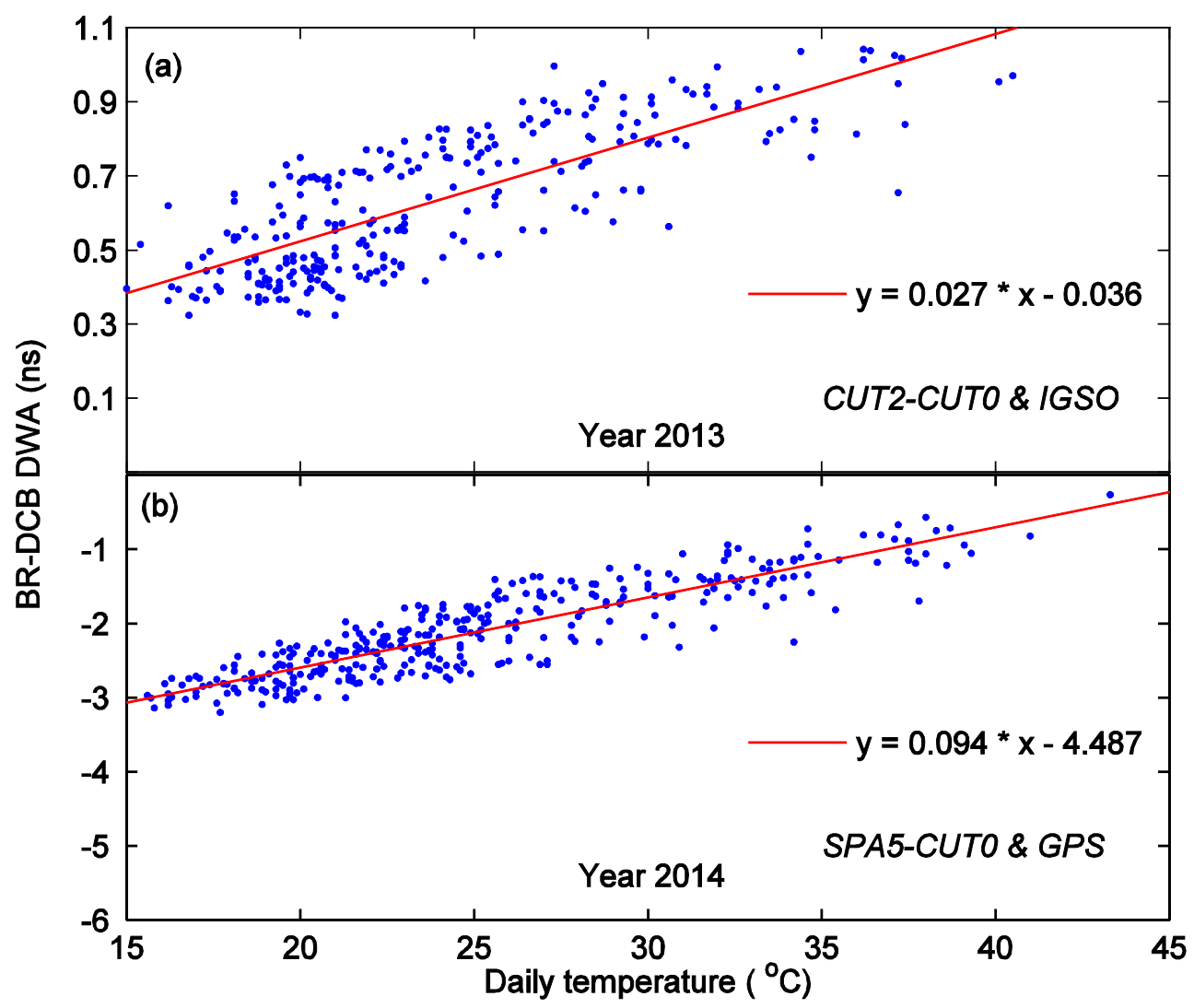

Fig. S4 Scatter plots graphically representing the same set of data that are shown in Fig. 4 using blue points. Each point has the values of daily temperature (horizontal axis) against BR-DCB DWA estimate (vertical axis). Superimposed on each scatter plot is the line of best fit in red. 


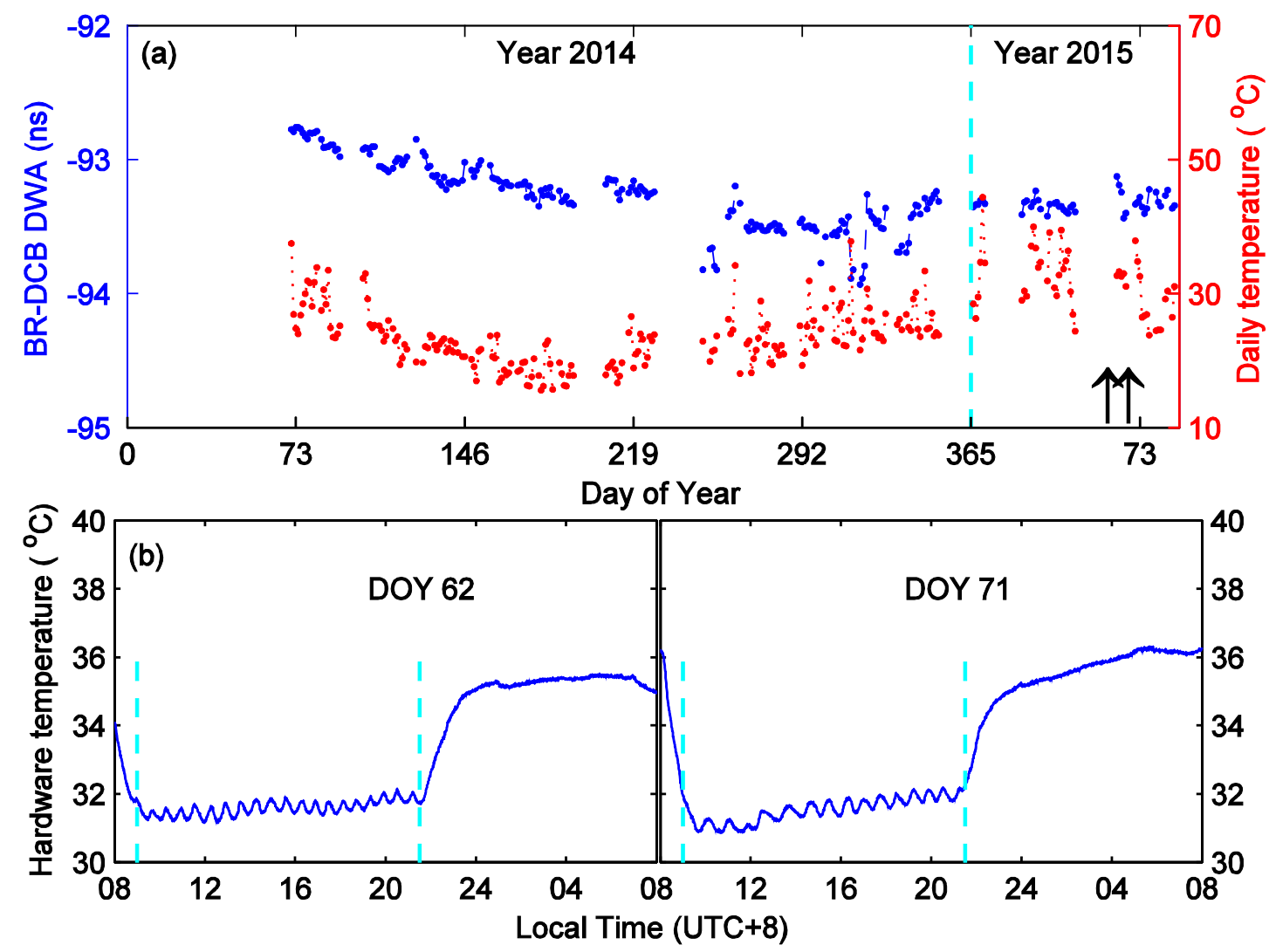

Fig. S5 The BR-DCB DWA estimates for receiver-pair SPA5-SPA7 (Trimble-Javad) and constellation BeiDou (GEO) (blue dotted line) versus daily maximum ambient temperatures (red dotted line) with PCC value of 0.01 (subplot a). The hardware temperatures with a 1-min time resolution for receivers SPA5 versus local time (subplot b): two vertical dash lines define the time interval from 9:00 am to 21:30 pm when the airconditioning system is turned on. 